\title{
Easing wave optics understanding through technology
}

\section{Amit Garg, Priyanka Kachru, Shatakshi Singh, Rishabh Tiwary}

Amit Garg, Priyanka Kachru, Shatakshi Singh, Rishabh Tiwary, "Easing wave optics understanding through technology," Proc. SPIE 9188, Optics Education and Outreach III, 91880T (15 September 2014); doi: 10.1117/12.2066379

EDIE Event: SPIE Optical Engineering + Applications, 2014, San Diego, California, United States 


\title{
Easing wave optics understanding through technology
}

\author{
Amit Garg, Priyanka Kachru, Shatakshi Singh, Rishabh Tiwary \\ Department of Electronics, Acharya Narendra Dev College, University of Delhi, Govindpuri, \\ Kalkaji, New Delhi-110019, India. \\ Email: agarg@andc.du.ac.in
}

\begin{abstract}
As part of the course curriculum of Physics of class XII, students do a comprehensive theoretical study about the wave nature of light specially related to interference, diffraction and polarisation. But, these studies are not backed up by any experiments. This makes the understanding of these complex topics very difficult. The purpose of the present outreach activity is to make students do many hands-on experiments on the above topics. The experiments have been designed keeping in mind the various theoretical concepts taught to the students. The studies are helpful in making the students understand fringe formation, intensity variation across the fringes formed helping them compare interference and diffraction fringes, dependence of fringe separation on various parameters, linear polarization, Malus' law and Brewster's law. The tools used to perform the experiments include He-Ne/ diode laser(s), Laptop/Digital Storage Oscilloscope, CCD, various optical components like set of polarisers and analysers, glass plate and hardware components like single slit and double slit. The class XII students are divided into batches and each batch is handled by a team of three University of Delhi at ANDC SPIE student chapter members. The gains of the activity are measured through pre and post-tests.
\end{abstract}

Keywords: Optics Outreach, Polarisation, Interference, Diffraction, CCD.

\section{INTRODUCTION}

In Physics, wave optics is branch of optics which does not prove to be in agreement with the approximations of geometrical optics. The fundamental contradiction between these two widely different fields of optics is that, one supports wave effects and full wave electromagnetism. With various implementations, Interference and diffraction are treated simultaneously as they both are effects of combining of waves by principle of superposition but we can say that concept of interference gives us the diffraction pattern with bending of light from the edges of the obstacle. By using the scalar theory, we avoid the vector nature of the electric field of the wave. Scalar diffraction theory allows us to treat mathematically the propagation of light and the effects of obstructions in its path [1]. The basis of almost all instruments for spectral analysis is again interference. Further, to produce multi-beam interference we introduce Fabry-Perot interferometry, and the division of wave-front is generalised by multiple slits in diffraction grating spectrometer. Now coming back to electric field vector, the direction of the electric field vector decides the polarisation. Hence, the light can be unpolarised, aligned in the linear direction; linearly polarised, or rotating in space; circularly or elliptically polarised light [1].

All these are the techniques to do the analysis of interference and diffraction patterns to unveil the outcomes incur exorbitant costs which undergraduate laboratories in colleges cannot support. Considering the limitations of the budget and expensive resources, students of SPIE Student Chapter at ANDC tried to improvise such cumbersome techniques through the use of CCD linear array. This could serve the purpose of a new innovation and also help in elevating the study of wave patterns which we frequently study and see only in our textbooks. After thorough discussions, this project was taken up by our SPIE Chapter mainly because wave optics is amongst very rare topics which are taken up as an outreach project and moreover it brings out a totally new concept about the propagation of light as a wave. Students with whom we will be dealing are of higher secondary level, and at this stage, they are little exposed to the very novel and

Optics Education and Outreach III, edited by G. Groot Gregory, Proc. of SPIE Vol. 9188 ,

91880T (C) 2014 SPIE · CCC code: $0277-786 X / 14 / \$ 18 \cdot$ doi: $10.1117 / 12.2066379$ 
completely mind-twirling concept of wave nature of light. At this stage, students are through with the ray optical phenomena and have built the basis of optics over its fundamentals, which are then violated by wave character of light at a microscopic level. Therefore, to smoothen the extent of understanding theory with the practical applications of wave optical phenomena at school level so that they don't face much difficulty while going about this transition, the wave optics demonstrations using CCD linear array were presented in schools to bridge the gap of their concepts.

\section{THEORY}

Through umpteen technologies focussing on improvement of previous outdated techniques for recording various results and extracting some desired information, laser interferometry has gained prominence as one of the recent breakthroughs in many areas of photonics. The cornerstone for these technologies involving interferometry is simply the fundamentals of wave optics. Interferometry derived from "interference" which is the first and foremost concept in wave optics. Wave optics being a vast field of research, is further subdivided into three branches: Interference, Diffraction and Polarisation.

\subsection{Interference}

The basic principle behind the interference pattern is the Huygen's Principle which states that all the points on the wave front of light may be regarded as sources of secondary wavelets that propagate in every direction.

Interference is observed by sending coherent light waves through two slits separated by a finite distance' $\mathrm{d}$ '. As a result we observe a series of alternate bright and dark bands of equal intensity and equal width. Let ' $x$ ' be the path difference a light must travel to project at a point on the screen from the two slits, ' $y$ ' be the distance of first bright/ dark fringe from the centre of the screen, ' 1 ' be the perpendicular distance between the screen and the slits[2].

As from the observations,

$$
\text { Width_of_fringes }=\lambda \frac{l}{d}
$$

For constructive interference (bright fringes):

$$
x=m \lambda
$$

For destructive interference (dark fringes):

$$
x=\frac{(2 m-1)}{2} \lambda
$$

From equation (1) we understand that as ' $d$ ' increases, the fringe width decreases and as ' 1 ' distance increases the fringe width increases.

Applications of light interference are used practically in interferometry to measure small distances of order of few wavelengths. Further its uses include, coating on windows to determine the precise thickness of the coating that minimises or maximises transmittance and reflectance [3]. Practical life applications include interference pattern observed with oil slick, antireflective glasses produces destructive interference used in spectacles.

\subsection{Diffraction}

Diffraction is the phenomena of bending of light from the edges of the obstacle or through the opening. The initial conditions for observing the diffraction pattern being that the size of the slit or the obstacle should be comparable to the wavelength of light used. So, any increase in the width of the slit will result eventually in the collapsing of the diffraction fringes at the centre.

Let ' $d$ ' be the width of the slit, ' $\lambda$ ' being the wavelength of light used. So we will get the equation for dark fringes:

$$
d \sin \theta=n \lambda
$$


Similarly, for bright fringes

$$
d \sin \theta=\frac{2 n-1}{2} \lambda
$$

Interference and diffraction are inter-related. As seen in the double slit experiment, if one of the slits is covered, the pattern changes to that of diffraction using singe slit. So, diffraction is the special case of Young's double slit experiment.

\subsection{Polarisation}

Polarisation is the property of waves in which the random components of electric field get aligned in one particular orientation. As when dealing with light, it is unpolarised which means that electric field vector is pointing in any direction until they are linearly aligned with introduction of a vertical polariser and oscillate in only one direction. This is the fact why light is said to be a transverse wave because it gets polarised.

As per Malus Law:

$$
I=I_{O} \cos ^{2} \theta
$$

According to equation (6), $\mathrm{I}_{\max }=\mathrm{I}_{0}$; for $\theta=0^{\circ}$

$$
I_{\text {min }}=0 \text {; for } \theta=90^{\circ}
$$

This is the case with only two polarisers. The further setup with three polarisers, with the second one aligned at $45^{\circ}$ and the others at $90^{\circ}$ with respective to each other, according to the formula, minimum intensity should be zero ( no transmission) but with the introduction of the third polariser, the minimum intensity has some non-zero intensity. It means that $45^{\circ}$ polariser changes the orientation of the linearly polarised light through first polariser and transmits it to the third polariser at $45^{\circ}$ resulting some intensity of the final wave.

The result can be verified by the following expression:

$$
\begin{aligned}
& I=I_{0} \cos ^{2}(45) \\
& I=I_{0}\left(\frac{1}{2}\right) \\
& I=\frac{I_{2}}{2}
\end{aligned}
$$

With this basic knowledge, linearly polarised can be converted into circularly polarised light by adjusting the plane of wave at $45^{\circ}$ with optic axis [4].

The implications of these fundamental laws are quite observable in daily life. Polaroid goggles are amongst the most common applications of polarisation. LCDs also use polarising filters. Apart from these everyday applications, the polarisation data of starlight is used to track the interstellar magnetic fields. In fact polarisation of cosmic background radiations helps us to know about the early universe [5].

\section{EXPERIMENTAL SETUP}

Our setup includes various components and devices which were readily available in our laboratory.

The list of equipment used during the course of this outreach activity is:

1. He-Ne Laser/ Diode Laser with voltage supply 
2. CCD (Charged Coupled Device) with USB port(2048 x 1 pixels)

3. Single slit

4. Double slits of different widths.

5. Two polarisers

6. Quarter wave plate

7. White distant screen

8. $\quad$ Slit holder and stands

\subsection{Diffraction pattern}

The experimental setup to study diffraction pattern due to a single slit with diode laser or He-Ne laser on a distant screen is as shown in Fig.1. After finally obtaining a perfect diffraction pattern, we project the pattern onto the linear CCD array which displays real time acquisition of intensity pattern on the laptop or computer configured for it (Fig.2).

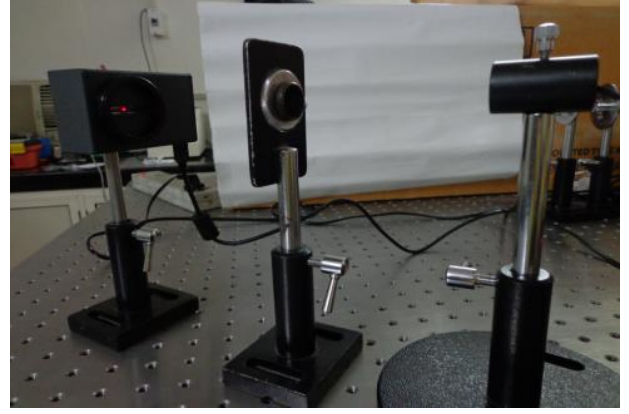

Fig.1: Diffraction pattern setup.

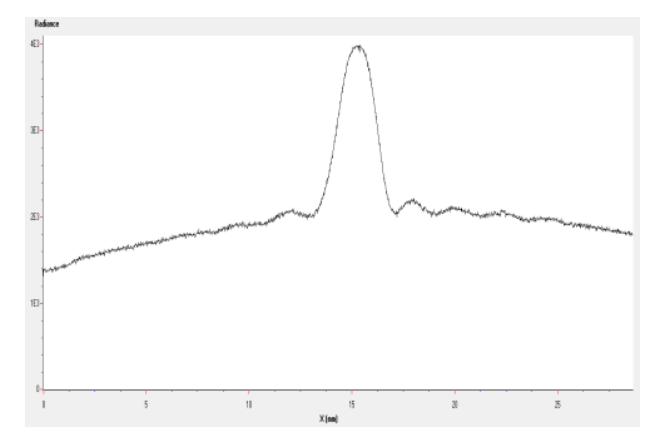

Fig.2: Pattern observed with CCD.

\subsection{Double slit Pattern}

We used double slits of different slit widths and observed the intensity patterns for each double slit. We replace the single slit with double slit grid and observe the patterns for different widths on the distant screen (Fig. 3). Similar to the diffraction pattern observed with linear CCD array, intensities of interference patterns are also obtained (Fig. 4-7). The resulting interference pattern has a diffraction pattern due to individual slits.

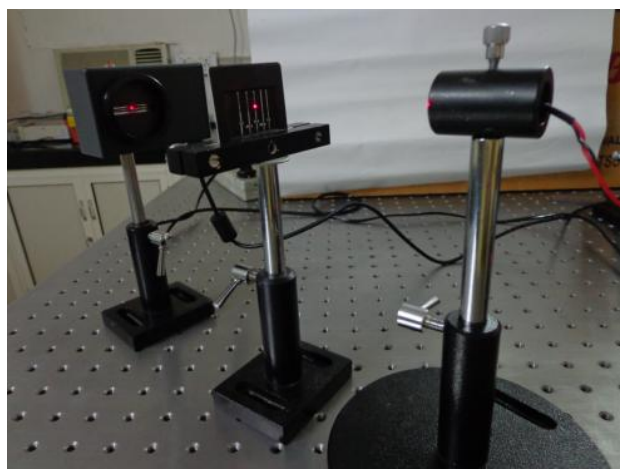

Fig. 3: Interference pattern setup.

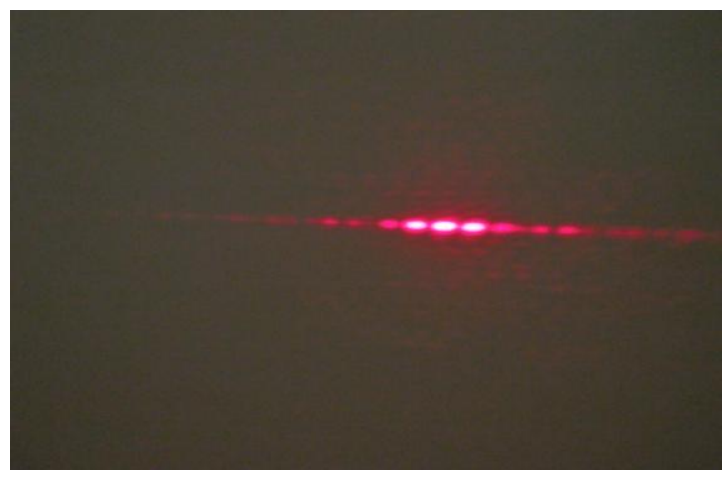

Fig. 4: Interference with $0.1 \mathrm{~mm}$ width double slit. 


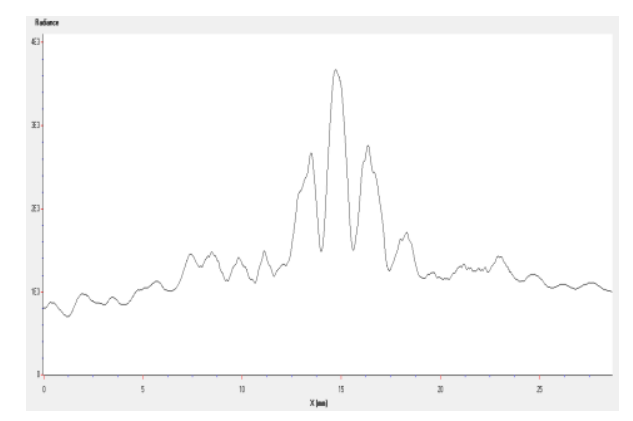

Fig. 5: Intensity pattern for $0.1 \mathrm{~mm}$ width slits.

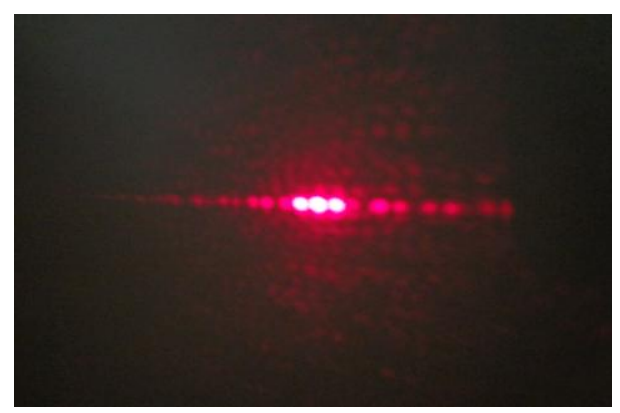

Fig. 6: Slits with $0.2 \mathrm{~mm}$ width

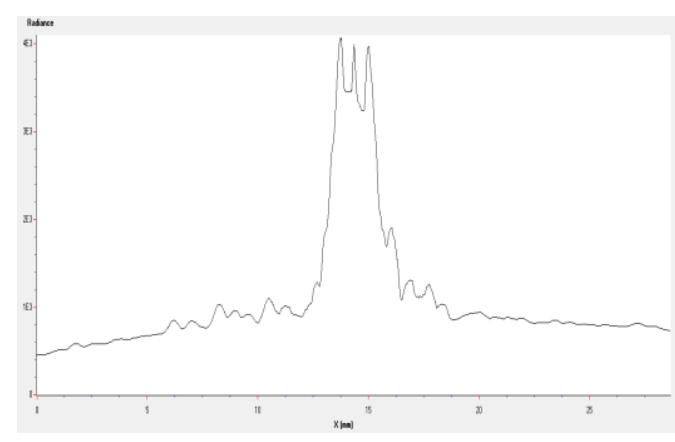

Fig. 7: Intensity on CCD for $0.2 \mathrm{~mm}$ width slits.

\subsection{Polarisation}

We align the laser source with a polariser and an analyser for the resultant intensity to be observed with linear CCD array (Fig. 8). Initially the polariser and analyser are set at zero degrees (parallel) to each other. As the angle increases (relative position changes from parallel to cross position), the intensity decreases according to the Malus law (Fig. 9, 10). Then we introduced a quarter wave plate between the two polarisers to explain the concept of elliptical and circular polarization. (Fig. 11, 12).

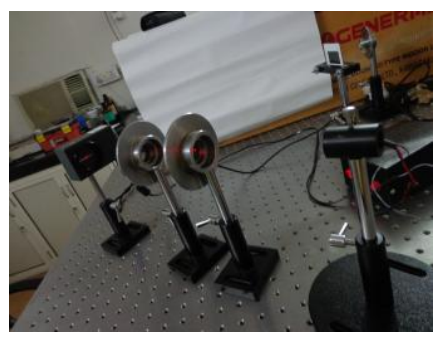

Fig.8: Polarisation setup for Malus law

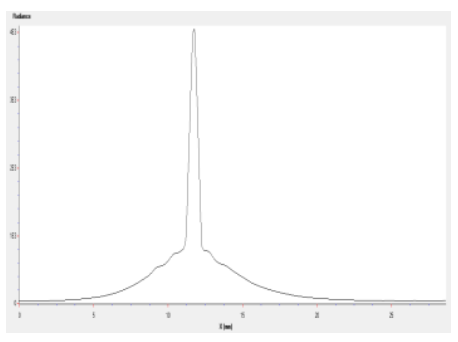

Fig. 9: Maximum intensity peak 


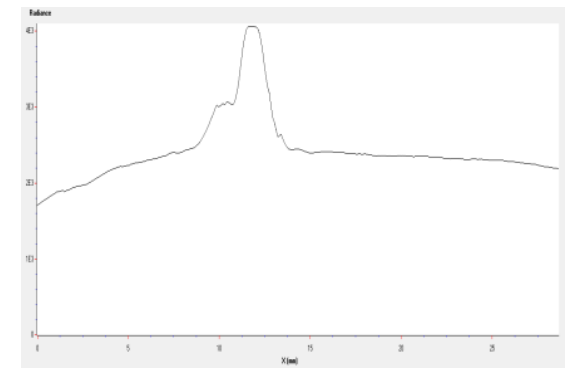

Fig. 10: Minimum intensity peak

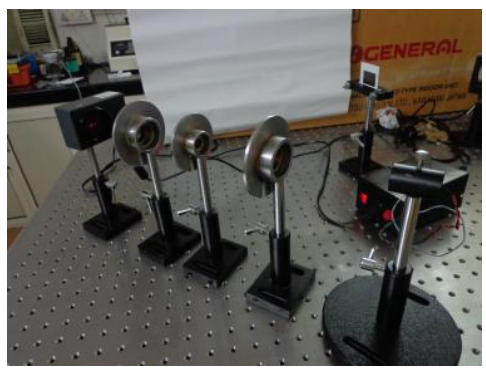

Fig. 11: Polarisation with quarter wave plate

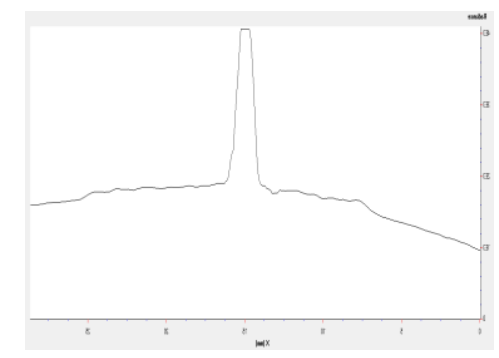

Fig. 12: Minimum intensity with quarter wave plate.

\section{METHODOLOGY}

Keeping the baseline of the above mentioned experiments quite simple and easy to grasp, we conducted this workshop in one of a reputed private school in Delhi. The workshop kick started with a lot of excitement and inquisitive gazes from the students onto our apparatus. As the students were not having any practical exposure to wave optics, so we had to set a bar for them in order to make them enjoy and learn equally from this workshop. For that, we had put up some basic questions to them so as to get clear idea of the level of their understanding at the start of the workshop.

From a ten minutes question and answer round, we concluded that some students had some vague idea about polarisation of light and knew about its very common application in Polaroid goggles. But still, they did not know the fundamental reasons behind these phenomenons. Since students have a little exposure to wave optics, we had to start from scratch and teach them all the rudimentary concepts of wave optics so as to enhance their understanding levels for our demonstrations. During the course of the workshop, students started with the interjections on every topic and as we proceeded towards the end of the workshop, they were all in awe of the demonstration on such a new concept which is quite briefly taught at school level. In fact, students were also giving us ideas on how to get polarised light using buffer solutions instead of polarisers. The best session throughout the workshop was the hands-on experience of students onto these experiments and let them have a closer look at how the experiments were done. Some common questions which were raised by the students were:

1. What is wave optics?

2. What is an electromagnetic wave?

3. How does light propagate in vacuum?

4. Why light is a wave?

5. Difference between ray optics and wave optics? 
6. What is a wave front?

7. What is constructive and destructive interference?

8. How is interference different from diffraction pattern?

All the teachers praised us for our hard work and a great innovation and students kept on asking us to come again for such a workshop because of the invoked interest due to hands-on exposure.

Fig. 13-18 shows some of the photographs taken during the workshop:

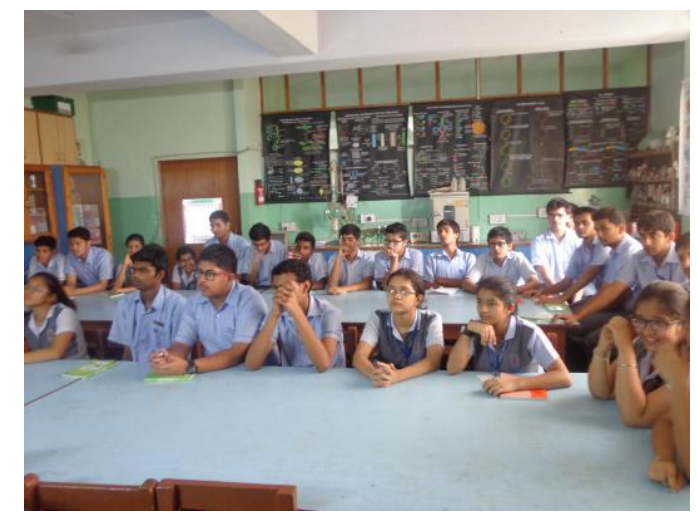

Fig. 13: Students engrossed in the workshop.

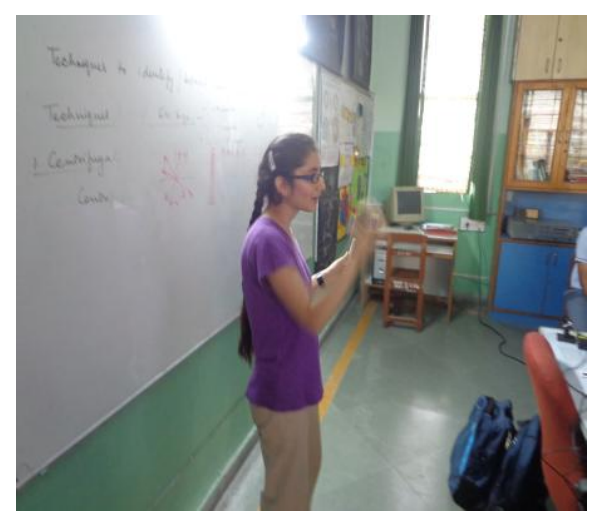

Fig. 14: Explaining wave optics to students.

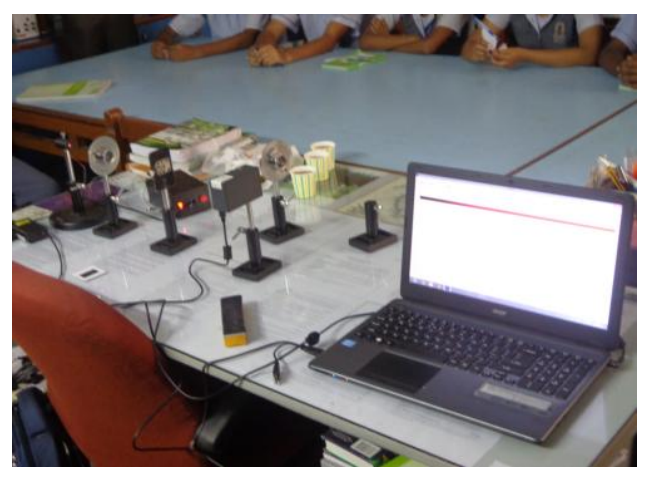

Fig. 15: Setup in school

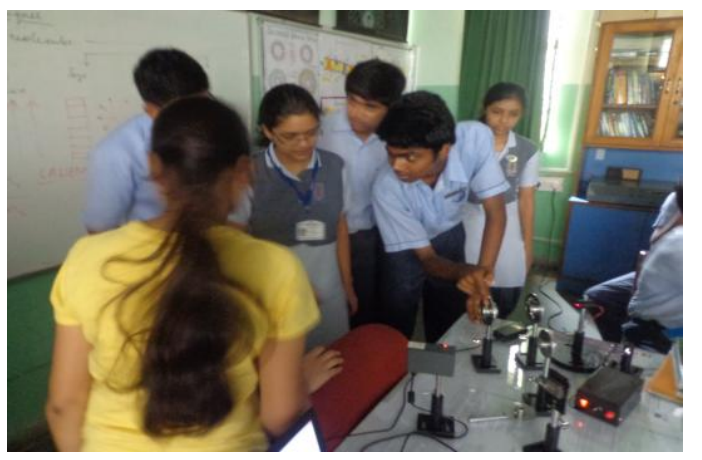

Fig. 17: Hands-on experience.

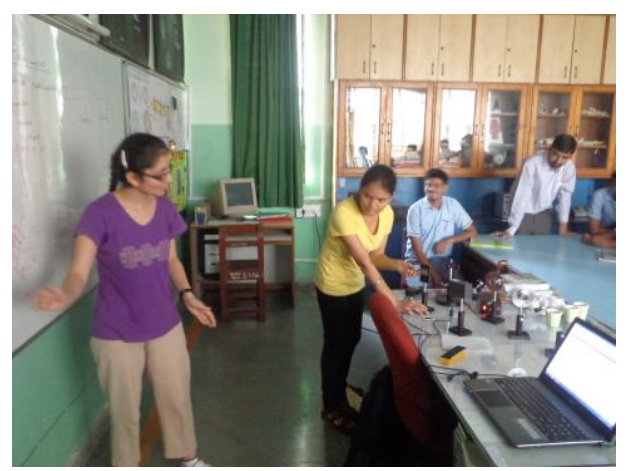

Fig. 16: Demonstrating diffraction

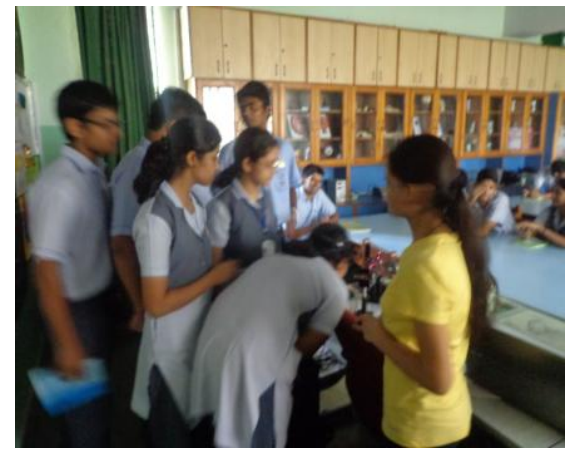




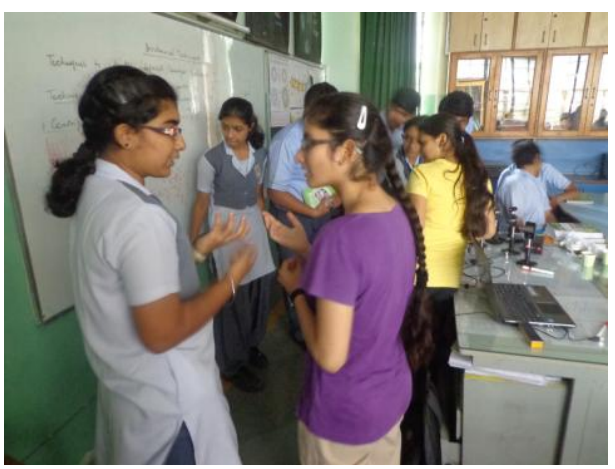

Fig. 18: Students sharing their ideas.

\section{RESULTS AND CONCLUSION}

The workshop concluded successfully with all the students applauding the innovations done and also bringing out some very minute aspects of wave optics which school curriculum doesn't support. Meanwhile, we also conducted pre and post test along with a feedback form to know students' opinion on the workshop and how much information they gained from such an activity and what improvement did they experience. Even the teachers did not hesitate in saying that students in schools get very rare opportunities to work hands-on onto such experiments which are only limited up to theories. Fig. 19 represents exhaustive analysis of the pre and post tests of the students. The results are very encouraging as the activity leads to vast improvement in their conceptual and application clarity. We plan to conduct many more such workshops in other schools of Delhi.

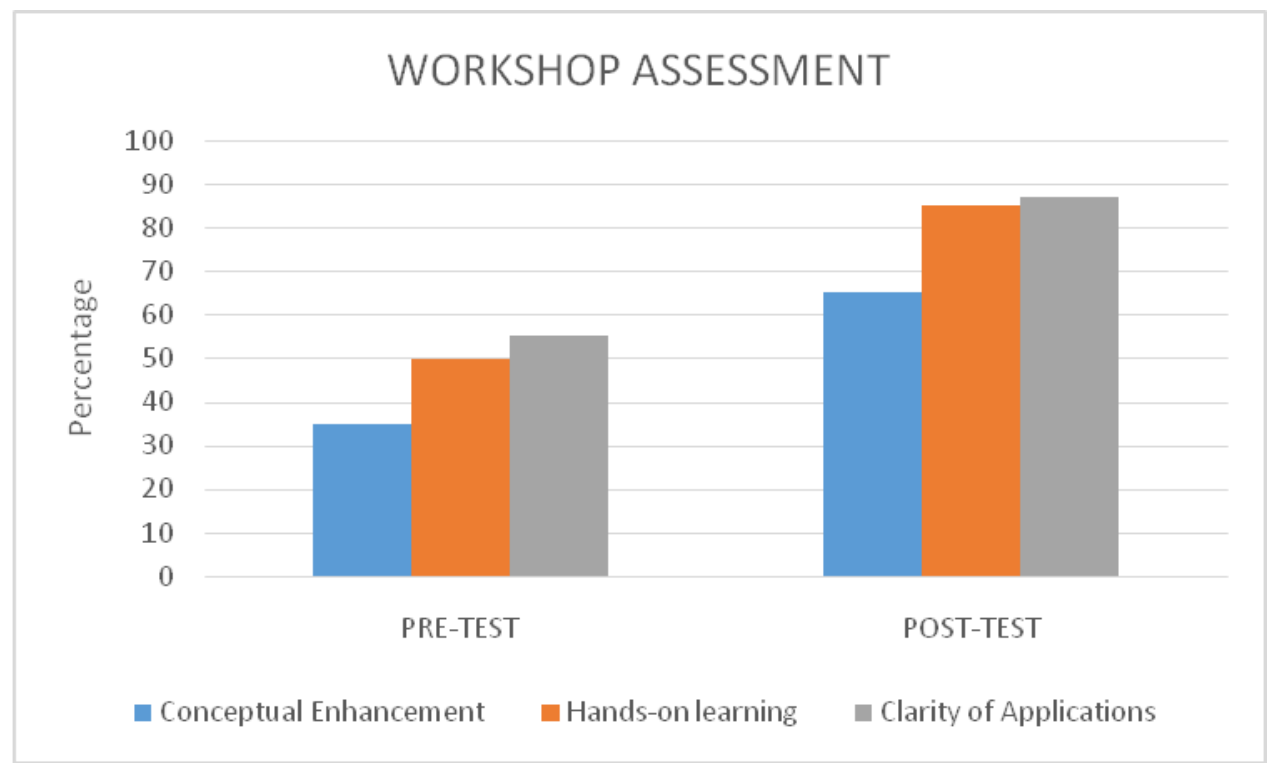

Fig. 19: Pre-test and Post-test analysis of the workshop.

Introduction to wave optics brought a vast side of optics which for class 12 students is only confined to ray optics. This workshop changed their perception about the behaviour of light as not only a ray but also has wave associated to it. 


\section{ACKNOWLEGDEMENT}

One of the authors, Priyanka Kachru expresses her sincere gratitude to SPIE, USA for awarding Officer's Travel Grant to attend and present this research paper in SPIE Optics+ Photonics 2014 conference from 17-21 August 2014 in San Diego, CA, USA.

\section{REFERENCES}

[1] P.Ewart, Optics: The Science of Light, $2^{\text {nd }}$ year Physics, pg.1 www.physics.ox.ac.uk/Users/smithb/website/coursenotes/A2optics_notes_2013.pdf

[2] Young's double slit experiment and Diffraction $\underline{\text { www.dev.physicslab.org/Document.aspx }}$ ?doctype=3\&filename=PhysicalOptics_InterferenceDiffraction.xml

[3] www.in.answers.yahoo.com/question/index?qid=20090427140811AAgNkDk

[4] www.hyperphysics.phy-astr.gsu.edu/hbase/phyopt/quarwv.html

[5] www.en.wikipedia.org/wiki/Polarization_in_astronomy 